\title{
CUMULENE CARBENES IN SPACE AND IN THE LABORATORY
}

\author{
J. M. VRTILEK, C. A. GOTTLIEB, \\ T. C. KILLIAN, and P. THADDEUS \\ Division of Applied Sciences \\ Harvard University \\ Cambridge, MA 02138, U.S.A.
}

\author{
J. CERNICHARO, M. GUÉLIN, \\ and G. PAUBERT \\ IRAM \\ Domaine Universitaire de Grenoble \\ F-38406 St. Martin D'Heres, France
}

\begin{abstract}
Astronomical searches for $\mathrm{H}_{2} \mathrm{CCC}$ and $\mathrm{H}_{2} \mathrm{CCCC}$, based on frequencies from our laboratory identifications, have resulted in detections toward TMC-1 and $\mathrm{IRC}+10216$. These new interstellar species are possibly the first of a new family of highly polar carbon chains; they are only the second and third carbenes (carbon molecules with two nonbonded electrons) known in space.
\end{abstract}

\section{Introduction}

As part of a long-term investigation of rotational spectra of transient and reactive molecules of astrophysical importance, we recently undertook a laboratory search for several members of a family of highly polar hydrocarbons with linear double-bonded carbon backbones and terminal nonbonded carbene electrons. Two of these, $\mathrm{H}_{2} \mathrm{CCC}$ and $\mathrm{H}_{2} \mathrm{CCCC}$, have now been successfully observed in the same cooled acetylene discharge that has been a source of numerous other reactive hydrocarbons (Vrtilek et al. 1990; Killian et al. 1990). They are remarkable for several reasons: both are isomers of interstellar species, $\mathrm{H}_{2} \mathrm{CCC}$ of cyclic- $\mathrm{C}_{3} \mathrm{H}_{2}$ and $\mathrm{H}_{2} \mathrm{CCCC}$ of diacetylene (almost certainly abundant though not directly detected); both are highly polar, with the two nonbonded electrons characteristic of carbenes; and, like a number of other transient interstellar species, both are unsaturated carbon chains.

With highly accurate $\left(\sim 1 \mathrm{~km} \mathrm{~s}^{-1}\right.$ or better in equivalent velocity) frequency predictions in hand, we initiated successful astronomical searches for $\mathrm{H}_{2} \mathrm{CCC}$ and $\mathrm{H}_{2} \mathrm{CCCC}$, using principally the IRAM $30 \mathrm{~m}$ telescope but also data from the Effelsberg $100 \mathrm{~m}$ and the NRAO $43 \mathrm{~m}$ (Cernicharo et al. 1991a,b). Observations were also obtained at Nobeyama (Kawaguchi et al. 1991).

Although the smallest $\mathrm{H}_{2} \mathrm{C}_{n}$ molecule, vinylidene, $\mathrm{H}_{2} \mathrm{CC}$, has proven elusive, larger members of this sequence, predicted to be stable and even more polar, are excellent candidates for laboratory and radioastronomical detection, and searches for them are now underway.

\section{Rotational Spectra of $\mathrm{H}_{2} \mathrm{CCC}$ and $\mathrm{H}_{2} \mathrm{CCCC}$}

$\mathrm{H}_{2} \mathrm{CCC}$ and $\mathrm{H}_{2} \mathrm{CCCC}$ are calculated (DeFrees and McLean 1986; Dykstra, Parsons, and Oates 1979 ) to be the isomers next in energy above their ground configurations. Very nearly prolate symmetric top molecules, with rotational spectra characterized by strong, harmonically related $R$ - 
branch lines, both species have many lines well situated for observation with $\mathrm{mm}$-wave and $\mathrm{cm}$ wave radio telescopes.

Our prediction of a very specific nearly harmonic sequence of doublets in the millimeter-wave rotational spectra of $\mathrm{H}_{2} \mathrm{CCC}$ and $\mathrm{H}_{2} \mathrm{CCCC}$ was the basis for a systematic laboratory search for these species. In ladders with $K_{a}>0$ the slight deviation from symmetry splits energy levels degenerate in the symmetric top case ( $K$-doubling). For intermediate values of $K_{a}$, here $K_{a}=3$, the doublet splitting takes on a distinctive and convenient signature of a few $\mathrm{MHz}$.

Support for our identifications comes from a number of chemical and physical assays, in addition to agreement of the observed spectrum with that expected for molecules with $\mathrm{C}_{2 v}$ symmetry and the geometry of $\mathrm{H}_{2} \mathrm{CCC}$ and $\mathrm{H}_{2} \mathrm{CCCC}$. For $\mathrm{H}_{2} \mathrm{CCC}$ we now have conclusive evidence: a full substitution structure (Killian et al. 1991, in prep.).

\section{Measurements in Space}

Our initial identification of $\mathrm{H}_{2} \mathrm{CCC}$ in TMC-1 rested on three lines found at the expected frequencies (Cernicharo et al. 1991a), from which we derived a rotational temperature of 4-6 K, consistent with expectations from other molecules, and a column density of $(2.5 \pm 0.5) \times 10^{12} \mathrm{~cm}^{-2}$, smaller by a factor of $\sim 70$ than that of the lowest energy isomer, cyclic- $\mathrm{C}_{3} \mathrm{H}_{2}$. Three lines detected toward IRC+10216 imply a rotational temperature of $25 \mathrm{~K}$ and a column density of $\sim 2.6 \times 10^{12} \mathrm{~cm}^{-2}$.

Eight mm-wave transitions of $\mathrm{H}_{2} \mathrm{CCCC}$ have been assigned toward $\mathrm{IRC}+10216$; all are fit by a single rotational temperature, $20 \pm 3 \mathrm{~K}$; the implied column density is $(1.6 \pm 0.4) \times 10^{13} \mathrm{~cm}^{-2}, 6$ times that of $\mathrm{H}_{2} \mathrm{CCC}$ toward IRC+10216 (Cernicharo et al. 1991b). Several lines of $\mathrm{H}_{2} \mathrm{CCCC}$ have now been found in TMC-1, as for $\mathrm{H}_{2} \mathrm{CCC}$ at precisely the expected frequencies (Kawaguchi et al. 1991). The column density of $\mathrm{H}_{2} \mathrm{CCCC}$ in TMC-1, $\sim 8 \times 10^{12} \mathrm{~cm}^{-2}$, is greater, as in IRC+10216, than that of the smaller molecule $\mathrm{H}_{2} \mathrm{CCC}$.

The presence of three closely spaced, low-lying lines, two ortho $\left(2_{12}-1_{11}\right.$ at $17.79 \mathrm{GHz}$ and $2{ }_{11}-1_{10}$ at $\left.17.94 \mathrm{GHz}\right)$ and one para $\left(2_{02}-1_{01}\right.$ at $\left.17.86 \mathrm{GHz}\right)$, in the spectrum of $\mathrm{H}_{2} \mathrm{CCCC}$ at frequencies conveniently located for radio astronomy permits accurate measurement of the orthopara ratio, hence a possible constraint on proton density, and a search in the two ortho lines for the Townes-Cheung effect found in $\mathrm{H}_{2} \mathrm{CO}$.

\section{References}

Cernicharo, J., Gottlieb, C. A., Guélin, M., Killian, T. C., Paubert, G., Thaddeus, P., and Vrtilek, J. M. 1991a, Ap. J. Letters, 368, L39.

Cernicharo, J., Gottlieb, C. A., Guélin, M., Killian, T. C., Thaddeus, P., and Vrtilek, J. M. 1991b, Ap. J. Letters, 368, L43.

DeFrees, D. J., and McLean, A. D. 1986, Ap. J. Letters, 308, L31.

Dykstra, C. E., Parsons, C. A., and Oates, C. L. 1979, J. Am. Chem. Soc., 101, 1962.

Kawaguchi, K., Kaifu, N., Ohishi, M., Ishikawa, S., Hirahara, Y., Yamamoto, S., Saito, S., Takano, S., Murakami, A., Vrtilek, J. M., Gottlieb, C. A., Thaddeus, P., and Irvine, W. M., 1991, Pub. A. S. J., in press.

Killian, T. C., Vrtilek, J. M., Gottlieb, C. A., Gottlieb, E. W., and Thaddeus, P. 1990, Ap. J. Letters, $365, \mathrm{~L} 89$.

Vrtilek, J. M., Gottlieb, C. A., Gottlieb, E. W., Killian, T. C., and Thaddeus, P. 1990, Ap. J. Letters, 364, L53. 